\title{
Objetos virtuales de aprendizaje para ciencias de la salud
}

M. ${ }^{a}$ Elisa de Castro Peraza ${ }^{a}$, Naira Delgado Rodríguez ${ }^{b}$, Francisco Javier Castro Molina ${ }^{\mathrm{c}}$, Nieves Doria Lorenzo Rocha ${ }^{\mathrm{d}}$, Jesús Miguel Torres Jorge ${ }^{\mathrm{e}}$, Adrián Guillermo de Vega de Castrof ${ }^{\mathrm{f}}$, Marcos Alonso Quintana ${ }^{\mathrm{f}}$ Jesús Manuel García Acosta ${ }^{\mathrm{c}}$

a Escuela Universitaria de Enfermería $\mathrm{N}^{\mathrm{a}} \mathrm{S}{ }^{a}$ Candelaria, Universidad de la Laguna-Servicio Canario de Salud, Directora, mcastrop@ull.edu.es , ${ }^{\mathrm{b}}$ Facultad de Psicología Universidad de La Laguna. Profesora, ndelgado@ull.edu.es, ${ }^{\mathrm{c}}$ Escuela Universitaria de Enfermería $\mathrm{N}^{\mathrm{a}} \mathrm{S}^{\mathrm{a}}$ Candelaria, Profesores, fcastrom@ull.edu.es, extjgarciaa@ull.edu.es , ${ }^{\mathrm{d}}$

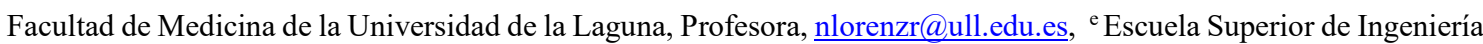
y Tecnología de la Universidad de la Laguna. Profesor, jimtorres@ull.edu.es y f Proyecto ENF19/17 FIISC, Becarios, adridevega@gmail.com, srkeitaro666@gmail.com.

\section{\$EWWFW}

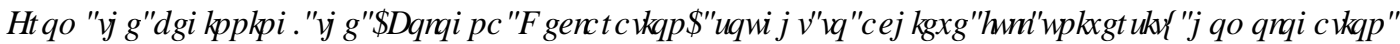

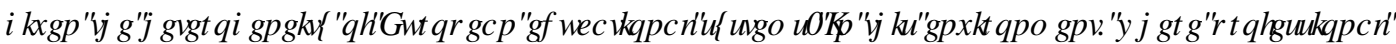

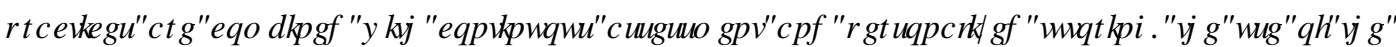

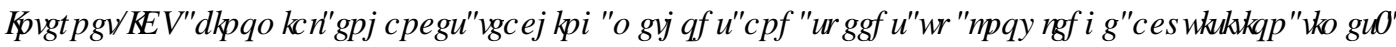

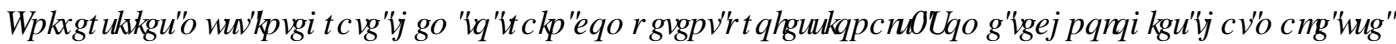

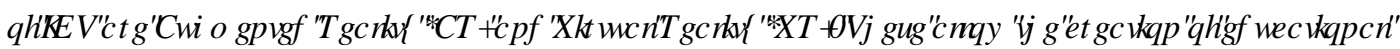

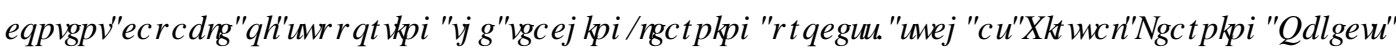

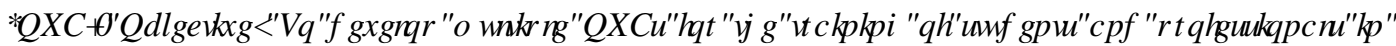

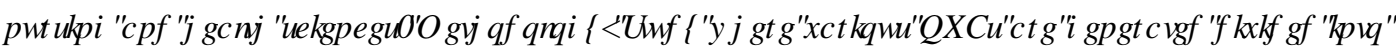

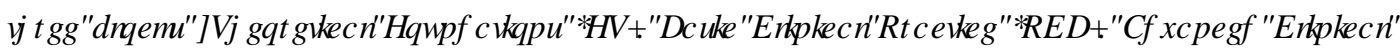

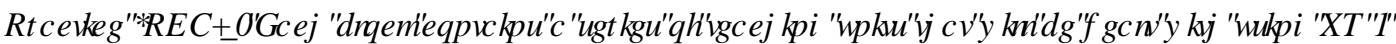

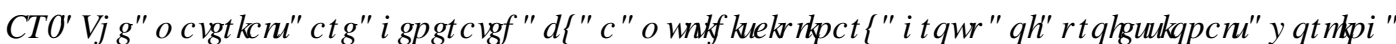

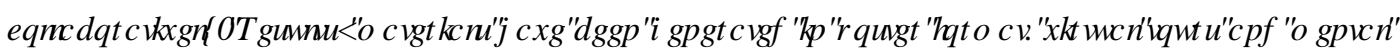

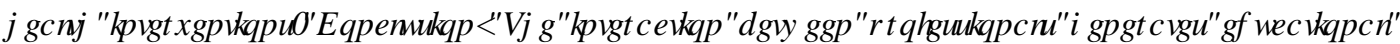

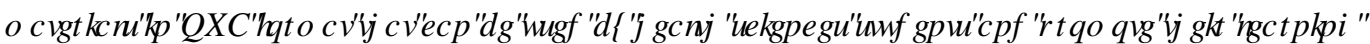

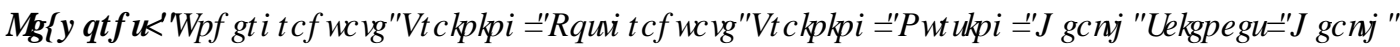

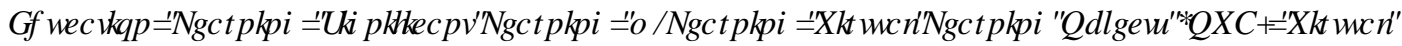
5 HDOWID XJP HQUAG55 HDOWD

\section{Resumen}

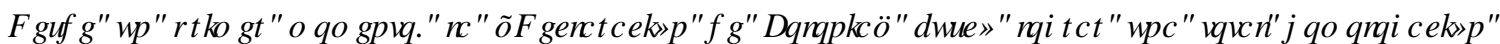

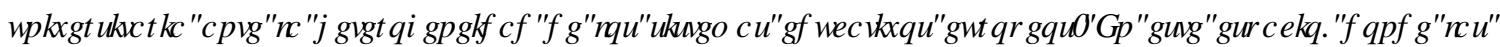

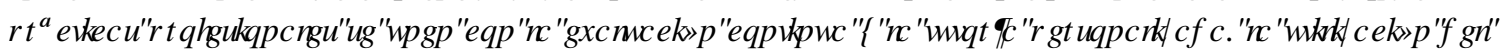

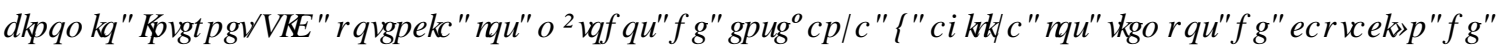

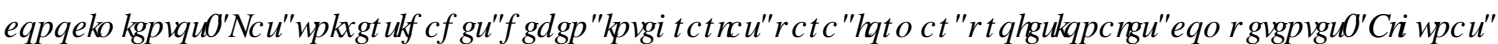

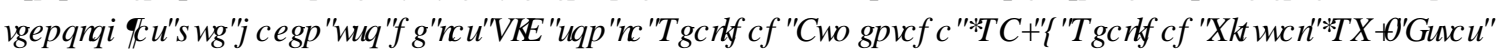

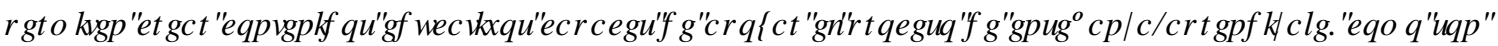

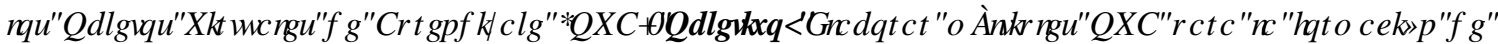

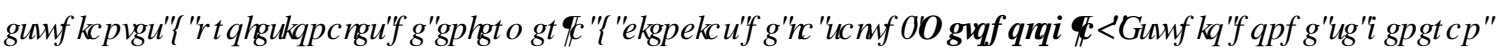




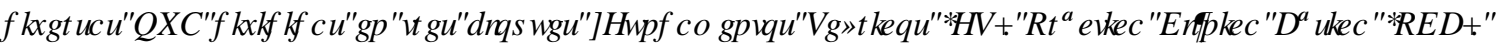

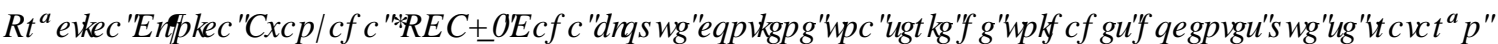

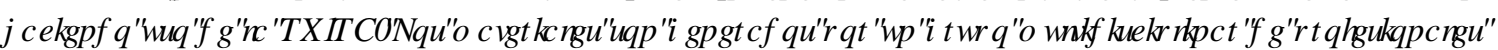

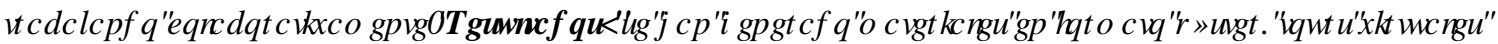

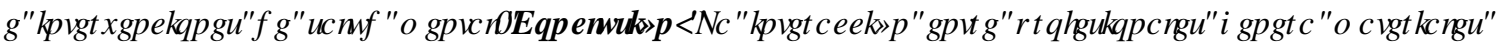

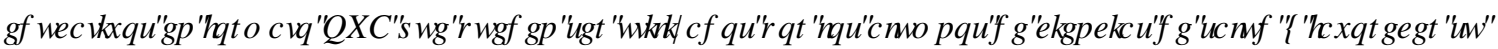
DSLHQG]DNAII

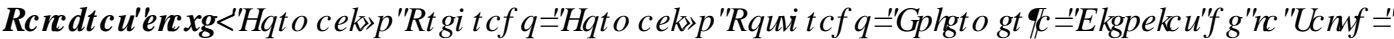

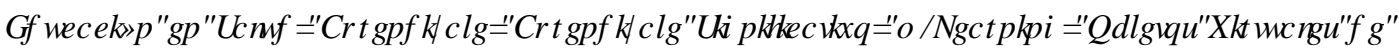

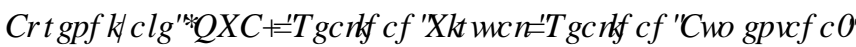

\section{Introducción}

La "Convergencia Europea en la Universidad" es un proceso producto de numerosas declaraciones periódicas entre las que destaca la de Bolonia (1999), en la que 29 países consensuaron una postura única bajo la "Carta Magna de las Universidades Europeas" de 1988. Se firmó el compromiso de crear un Espacio Europeo de Educación Superior (EEES) con el objetivo de lograr una armonización de los diferentes sistemas universitarios, buscando homogeneizar la estructura de los estudios, compartiendo la orientación y lo que algunos autores han denominado "economía del conocimiento" (Trujillo, 2009).

Desde un primer momento, la "Declaración de Bolonia" mantuvo el compromiso de lograr una total homologación de los sistemas universitarios, teniendo presente la heterogeneidad de los sistemas educativos europeos. Con este objetivo, se definió el valor académico único, el "European Credit Transfer System" (ECTS), ya empleado con anterioridad en el programa Erasmus. A ella siguieron otros comunicados como el de Praga (2001), Goteborg (2001), Berlín (2003) firmada por los ministros de educación de 33 países europeos bajo el lema "Realizando el espacio europeo de educación superior", Bergen (2005) y Londres (2007) (Corcó, 2008).

La creación del EEES estableció como objetivos fundamentales los siguientes: adopción de un sistema para comparación de titulaciones; reformulación de la educación en torno al aprendizaje del alumno y desde la óptica de la transmisión de contenidos; reconocer y fortalecer la movilidad de alumnos y profesores, alentar la competitividad en investigación y la formación continua; formular criterios académicos, competenciales y de cualificación profesional que se ajusten a la demanda del mercado laboral europeo; y adopción de un sistema vertebrado en pregrado y posgrado, con un planteamiento de títulos, contenidos curriculares y materias con valor en el mercado de trabajo. Este último, con la finalidad de la incorporación al mundo laboral con la formación más apropiada (Real Decreto 55/2005 de 25 de enero), promoción de la movilidad, fomento de la cooperación europea para garantizar la calidad de la educación superior y promoción de una dimensión europea de la educación superior (Nicol, 2005).

En 2006, la Comisión Europea propuso crear el "Marco Europeo de Cualificaciones" (MEC), con el fin de establecer un sistema de comparación de cualificaciones y niveles. Entre sus objetivos destaca fomentar el aprendizaje permanente y la movilidad geográfica en el mercado laboral. Este nuevo enfoque permitió una cooperación en territorio europeo en el campo de las cualificaciones sin precedentes, instaurando descriptores que tomaban como referencia los resultados de aprendizaje. La finalidad de su creación fue favorecer la comparación de cualificaciones entre los diferentes países miembros (Rué, 2007). 
El MEC, en la actualidad, permite la clasificación de niveles y sistemas de cualificación. Para la definición y organización de los resultados se distingue entre conocimientos, destrezas y competencia (CDC), como el fundamento de dicho marco. El MEC es un "marco de cualificaciones basado en los resultados de aprendizaje" en el que los descriptores abarcan todos los resultados de aprendizaje. Su distribución en ocho niveles le otorga el calificativo de escala, volviéndose más complejo en los niveles más elevados (Yániz, 2008).

Pero la Convergencia Europea en la Universidad supuso mucho más que crear un espacio europeo de educación superior de calidad, con un sistema de créditos que facilita la movilidad de los estudiantes, profesores e investigadores. Tras la reunión de Londres (2007), aparece una referencia explícita al cambio de modelo, del centrado en el profesor al centrado en el aprendizaje del estudiante. Con este modelo, se buscaba promover el aprendizaje del alumno, su autonomía y sus habilidades de autorregulación e incorporaba la "enseñanza innovadora", con diferentes métodos: trabajo cooperativo, aprendizaje basado en problemas, desarrollo de proyectos, etc. (López, 2017).

Así, el EEES genera un nuevo modelo docente con dos fundamentos claros: enseñanza práctica, en la que el alumno mantiene una intervención activa gracias al trabajo en grupo, las prácticas profesionales o los ejercicios, entre otros; y evaluación continua, donde la clave radica en el seguimiento diario del trabajo del alumno, por medio de la tutoría personalizada y el binomio Internet y nuevas tecnologías TIC (Trujillo, 2019; Sanabria, 2019).

La utilización de las TIC potencia los métodos de enseñanza y agiliza los tiempos de captación de conocimientos, por lo que las universidades tienen la oportunidad de integrarlas en los procesos de enseñanza - aprendizaje para formar profesionales competentes. El rápido progreso de las TIC transforma continuamente el modelo de búsqueda, selección, producción y transmisión de los conocimientos. Los sistemas educativos no pueden estar ajenos a esta realidad y para adaptarse a una sociedad en la que cada vez las TIC se encuentran más integradas, deben renovar tanto sus métodos pedagógicos como sus contenidos. Actualmente, la curiosidad es una de las características que impulsa el aprendizaje. Así, la calidad de la enseñanza demanda la incorporación de recursos y estrategias diversas para crear en el aula un ambiente de aprendizaje participativo, colaborativo, práctico y ameno.

Algunas de las tecnologías que hacen uso de las TIC son los dispositivos móviles y la Realidad Aumentada (RA) y Realidad Virtual (RV), los cuales permiten crear contenidos educativos capaces de apoyar el proceso de enseñanza-aprendizaje, como son los Objetos Virtuales de Aprendizaje (OVA). Los OVA son estructuras organizadas y diseñadas por equipos multidisciplinares que usan las ventajas de la RA/RV para captar la atención del público al cual va dirigida la enseñanza (López, 2017). Entendemos por RA la combinación de elementos virtuales sobre el mundo real, mientras que la RV se desarrolla sobre un entorno totalmente inmersivo. La RA y la RV están separadas, pero estrechamente relacionadas con las tecnologías. La RA se caracteriza por la incorporación de información digital incluyendo imágenes, vídeo y audio en el mundo real. La RA pretende mezclar la realidad con lo virtual, lo que permite a los usuarios interactuar con los dos objetos, el físico y el digital. La RV permite a los usuarios el paso a un mundo alternativo de inmersión, simulado por ordenador (Johnson, 2016). La RV es una herramienta que favorece la curiosidad en un entorno controlado donde probar y aprender-haciendo (Calderón, 2019).

Los equipos que soportan las TIC están cambiando los contenidos, la forma de transmitir y recuperar la información y la manera de convertirla en conocimiento. La primera revolución se produjo con la inclusión de los ordenadores en el ámbito de aprendizaje tradicional: el e-learning; pero la llegada de los sistemas de compresión ha permitido trabajar con equipos fuera del aula, creando un modelo formativo que rompe 
espacios, tiempos, tipos de usuarios y socializa de manera universal. Este proceso configura un nuevo modelo de enseñanza-aprendizaje basado en dispositivos digitales móviles: el m-learning. El m-learning apoya el aprendizaje significativo, dado que su portabilidad permite a los estudiantes realizar consultas en el propio lugar donde se necesite y favorece el modelo de aprender haciendo (Cadavieco, 2017).

Son múltiples las investigaciones que denotan el éxito académico derivado directamente del uso de la $\mathrm{RA} / \mathrm{RV}$, puesto que provocan un mayor rendimiento en el aprendizaje del alumnado. La RA/RV es una puerta abierta a nuevos mundos, al desarrollo de la imaginación y al incremento de la creatividad (Johnson, 2016). Pero quizá el elemento descrito de una manera más amplia es el elevado potencial que tiene la RA/RV como motivador de la formación (Sanabria, 2019; Ruiz Cerrillo, 2019; Calderón 2020).

El m-learning inmersivo es una nueva manera de educación. Dentro de este fenómeno, la RA/RV provoca una interacción con el estudiante en un mundo virtual en el que se siente inmerso y donde convive con videos e imágenes $3 \mathrm{D}$, textos, vínculos a internet, etc. Todos estos mundos pueden ayudar a comprender mejor los contenidos propuestos. Los entornos digitales inmersivos enriquecen y explican la realidad y lo hacen configurando espacios que no podrían reproducirse en el aula, tales como espacios microscópicos, peligrosos, restringidos, astronómicos, etc. (Cadavieco, 2017).

\section{La realidad virtual y motivación para el aprendizaje en ciencias de la salud}

La práctica de la medicina y de la enfermería tiene actualmente algunos aspectos que la diferencian de etapas anteriores. Entre las principales diferencias encontramos el trabajo en equipos multidisciplinares, la simultaneidad de múltiples datos de distintas fuentes, la toma de decisiones complejas y con altos niveles de incertidumbre, la necesidad de poner en marcha competencias diferentes que deben converger en una misma acción y la participación activa del paciente en la toma de decisiones que le atañen. A esto se le añade la incorporación de las TIC, los cambios culturales y hasta una nueva realidad social: cada vez son más los pacientes crónicos y los pacientes frágiles por edad (Ruiz Cerrillo, 2019). Esto obliga a buscar enfoques distintos en las ciencias de la salud que incluyan el replanteamiento de los profesionales y la necesidad de un reciclaje continuo. Las competencias van más allá de las clínicas puras, siendo necesarias habilidades de comunicación y un buen manejo de las TIC. Para poder alcanzar este perfil, el alumno debe de ser, desde el primer momento, el propio protagonista de su formación; relegando la función del profesor a la de tutor.

La simulación de experiencias clínicas es un método facilitador de habilidades clínicas en escenarios similares a la realidad (Calderón, 2020). El avance de la informática y la bioingeniería, entre otras, ha propiciado la mejora de los escenarios dedicados a la inmersión de los individuos en mundos virtuales que recrean el escenario clínico real. La RV como factor dinamizador de la conducta, propicia suficiente interés y motivación como para que los alumnos mejoren su actuación individual y grupal, incrementando la motivación y la autoestima (Calderón, 2020). Por ello, muchas instituciones educativas tratan de implementar experiencias de RV/RA, especialmente en profesiones como las sanitarias, que encuentran múltiples beneficios de una interacción profesional-paciente simulada. De este modo, el alumno puede experimentar técnicas sanitarias sin miedo a equivocarse y a causar un daño o una lesión. También puede aprender a moverse en entornos altamente complejos y demandantes antes de llegar "en el mundo real" a tener que trabajar en ellos. Por otra parte, la relación entre estudiantes y pacientes en los servicios hospitalarios está sujeta a la orden SSI/81/2017, de 19 de enero, donde se aprueba el protocolo mediante el que se determinan pautas básicas destinadas a asegurar y proteger el derecho a la intimidad del paciente por los alumnos y residentes en Ciencias de la Salud (Bolentín Oficial del Estado, 2017). En esta orden se establece que los pacientes tienen derecho a saber que hay alumnos en formación presentes en su proceso 
asistencial, y que deben autorizar su presencia. Además, se establece que, salvo supuestos especiales consentidos por el paciente, no deberán estar presentes más de tres alumnos por paciente. También la repetición de actividades asistenciales con grupos diferentes está sujeta a planificación para garantizar que no resulte molesta o intimidatoria para el paciente. Estas limitaciones de acceso a la realización de procedimientos clínicos justifican la necesidad de acercar la realidad asistencial al estudiantado a través de fórmulas complementarias al desarrollo de las prácticas clínicas.

Un estudio de revisión sobre el uso de la realidad simulada como estrategia de enseñanza en enfermería (Medina, 2017) revela interesantes consideraciones. Según sus autores, las principales ventajas de la realidad simulada en la educación son: desarrollo de la autoconfianza, incentivo de trabajo en equipo, aumento de habilidades de pensamiento crítico, ambiente controlado y seguro, retroalimentación inmediata sobre lo realizado, experiencia de aprendizaje interactiva a articulación de la teoría con la práctica clínica. Sin embargo, también presenta algunas desventajas que es importante tener en cuenta. Entre ellas, destaca la necesidad de una inversión económica, así como de tiempo y recursos, incluido un personal competente para usar, cuidar y mantener esta realidad simulada (Medina, 2017).

\section{Implementación de la RV/RA en situaciones concretas de salud}

Una de las aplicaciones de RV que se llevan más tiempo usando es en el tratamiento de las alteraciones de la imagen corporal que se suelen asociar con los trastornos de la conducta alimentaria. El constructo Imagen Corporal tiene tres componentes: el perceptivo que se refiere a la exactitud con que la persona estima sus dimensiones; el cognitivo que se refiere a los pensamientos y creencias sobre el cuerpo y las experiencias corporales y el emocional que son las experiencias de satisfacción/insatisfacción con la apariencia o experiencia corporal (Martín Garcés, 2019). Esto hace que las alteraciones de la imagen corporal deban de ser consideradas desde varios aspectos, todos ellos se benefician de la RV. A raíz del desarrollo de las TIC y la RV se generan experiencias pioneras con el objetivo de la recreación de ambientes virtuales en el estudio y tratamiento de las alteraciones de la imagen corporal. Muchos estudios han seguido esta estela, siendo los trastornos de la conducta alimentaria un campo de acción importante para los profesionales de la salud haciendo uso de la RV (Martín Garcés, 2019).

Otra de las situaciones clínicas susceptibles de mejora con el uso de la RV/RA es el manejo del dolor. El dolor se ve fuertemente influenciado por la capacidad del paciente para evadirse y no concentrarse en el estímulo que lo produce. Por ello, la distracción favorece el control del dolor, ya que los procedimientos de distracción propician la competencia entre estímulos y cargan la limitada capacidad atencional del paciente. Un trabajo de revisión (Crespo, 2020) sobre el uso de realidad virtual para el manejo del dolor en contextos clínicos, revela que la RV se puede aplicar de forma efectiva y segura para facilitar la reducción del dolor en un amplio rango de problemas y procedimientos médicos. Incluso las investigaciones de laboratorio en las que el dolor es inducido a personas sanas que participan voluntariamente, confirman la utilidad de la RV para su control (Crespo, 2020). Derivado del manejo del dolor surge también un estudio que analiza la eficacia de la RV como método de distracción no farmacológica durante la técnica de canalización de vía venosa periférica en un servicio de urgencias de pediatría. Sus resultados revelan que la RV es una herramienta eficaz para disminuir los niveles de dolor y ansiedad durante la punción venosa en pacientes pediátricos. Además, los padres se muestran más confortables tras ver cómo disminuye el dolor en sus hijos (Valbuena, 2019).

Una última derivada compleja del manejo del dolor es el dolor del miembro fantasma. Este dolor aparece en aproximadamente el $80 \%$ de los pacientes amputados. En muchos casos es un dolor intenso y constante 
que disminuye su calidad de vida. Con estos pacientes se ha utilizado la denominada "Terapia del Espejo", que consiste en la colocación de un espejo vertical en el plano sagital del paciente donde puede reflejarse el miembro sano. La ilusión visual del movimiento del miembro afectado genera una retroalimentación positiva a la corteza motora, lo que podría a su vez interrumpir el ciclo del dolor (Badía, 2018). La realidad virtual podría usarse con pacientes amputados de manera muy prometedora. La RV detecta la posición y los movimientos de la extremidad intacta y transfiere la imagen al espacio de la extremidad perdida. De esta manera el paciente puede "visualizar" una extremidad que ya no está, activando esa misma retroalimentación positiva. Las ventajas de utilizar RV frente a la terapia del espejo son que permite mayor inmersión y concentración para percibir la ilusión como real y centrar el movimiento, enfocándose mejor en la zona afectada, y mejorando por tanto su efectividad en la reducción del dolor a largo plazo (Badía, 2018).

Dentro de los usos de la RV en el ámbito de la salud, uno de los que más se puede beneficiar es el tratamiento psicológico. El bloque de tratamientos frente a las fobias tiene una amplia trayectoria. Las intervenciones psicológicas para estos problemas basadas en la terapia de exposición han demostrado ser eficaces, pero dada la naturaleza de las fobias tienen importantes limitaciones. La RV ayuda a superar estas limitaciones económicas, de tiempo, esfuerzos y oportunidad, ya que permiten recrear diferentes ambientes en los que la persona puede interactuar con sus miedos mientras está en un medio seguro y protegido (Viedman, 2017; Levy, 2018; Figuerola, 2018).

\section{Justificación del trabajo}

La Universidad de la Laguna (ULL) en Tenerife es una universidad pública cuyo fundamento docente está centrado en el alumno. De sus facultades de ciencias de la salud (entre otras: enfermería, medicina y psicología) egresan anualmente muchos nuevos profesionales que requieren estas competencias digitales en sus currículos. La ULL, igual que busca el aprendizaje significativo para sus alumnos, también requiere que sus profesionales trabajen de forma colaborativa para buscar metodologías centradas en el alumno y que impacten en su motivación para aprender y en su capacidad de ser profesionales de alto nivel. Especialmente en ciencias de la salud, esta meta es muy pertinente dado que se trata de la atención a personas en situaciones de vulnerabilidad por la enfermedad.

En este proyecto trabajan de forma colaborativa cuatro facultades: enfermería, medicina, psicología e informática. El objetivo es generar materiales educativos innovadores. En este sentido, la RA/RV y la creación de OVA supera cada vez más la faceta instrumental para convertirse en metodología educativa, con especiales opciones hacia el aprendizaje basado en el descubrimiento. Estos recursos permiten al usuario una inmersión en áreas como los fundamentos de la profesión, o áreas específicas o de acceso restringido (por ejemplo, quirófano, UVI, etc.).

Este proyecto se desarrolla en dos fases. La primera fase, a la que corresponde el trabajo que se presenta, tiene como objetivo generar una serie de OVA con contenidos específicos para ciencias de la salud. En la segunda fase del proyecto se comprobará si la utilización de herramientas digitales en formato OVA aumenta el nivel de adquisición de conocimientos y el grado de satisfacción de los usuarios. La segunda fase será probada por alumnos de la Escuela Universitaria de Enfermería $\mathrm{N}^{\mathrm{a}} \mathrm{S}^{\mathrm{a}}$ de Candelaria. Este centro, público y oficial, con un total de 240 alumnos por curso, egresa cada año a 60 profesionales de enfermería. Está considerado actualmente el primer centro de elección para estudiar enfermería dentro del panorama nacional, según la nota de corte. Pertenece tanto a la ULL como al Servicio Canario de la Salud. 


\section{Objetivos}

\section{Objetivo Principal:}

Elaborar materiales didácticos digitales para la formación de alumnos y profesionales de enfermería y de ciencias de la salud (en formato OVA y basados en RA/RV).

\section{Objetivos secundarios:}

Incorporar los materiales didácticos digitales elaborados en el plan de estudios de enfermería, dentro de las áreas de Fundamentos teóricos (FT), Práctica clínica básica (PCB) y Práctica clínica avanzada (PCA).

Desarrollar materiales a partir de situaciones y escenarios reales de práctica clínica o intervención, que permitan al alumnado tener experiencias muy próximas a las que se tienen en contextos reales.

\section{Desarrollo de la innovación}

En el marco de un proyecto de investigación, se generan seis OVAs de diferente profundidad y dificultad de contenidos en función del nivel al que vayan dirigidas: alumnos de primero, de segundo y alumnos avanzados o incluso profesionales como parte de su formación de postgrado. Concretamente, se generan tres bloques de contenidos (según el grado de dificultad y el nivel del alumno al que vayan dirigidos). Cada bloque contiene una serie de unidades docentes que se tratarán haciendo uso de la RV/RA, que buscan sustituir al esquema actual de clase magistral con visionado de diapositivas y vídeos convencionales.

Los profesionales que integran los diferentes grupos del proyecto son profesionales multidisciplinares, mayoritariamente de enfermería y ciencias de la salud, pero también se colabora con otras disciplinas como es la informática y la educación, para poder generar materiales innovadores.

El estudio se desarrolla en dos grandes fases:

En la primera fase se reúne un grupo de expertos y plantea las diversas áreas formativas, dentro del currículum del profesional de enfermería, que pueden ser abordadas con OVA y tratadas con RA/RV. Este grupo de expertos también genera las líneas maestras de cómo deben de ser los contenidos de cada OVA para que se ajusten de forma homogénea en todos los bloques del proyecto. A su vez, de este grupo de expertos salen una persona referente para la constitución de cada uno de los grupos focales que elaboran cada uno de los OVA seleccionados.

En la segunda fase tienen lugar las sesiones de los grupos focales para desarrollar cada uno de los OVA. Los contenidos explicados en estas unidades docentes han sido consensuados con todos los implicados en el estudio, trabajando de forma colaborativa en grupos focales multidisciplinares con participación de alumnado además de profesorado y profesionales. Esta fase se realiza en tres sesiones, una por semana tratando una unidad docente en cada uno de los grupos focales. Cada sesión explora y decide el contenido de cada OVA y los resultados de aprendizaje que se espera obtener con su aplicación. También se identifica la metodología actual para explicar ese contenido dentro del esquema formativo actual. Con ello, se pretende poder comparar ambas metodologías docentes (RA/RV frente a metodología convencional) en un estudio futuro. Con el objetivo de evitar el sesgo de selección entre el alumnado, se ha invitado a participar a todos los estudiantes. Esta actividad no lleva nota asociada que influya en la calificación del alumno.

De cada una de las reuniones de los grupos se crea un documento de consenso que servirá para hacer un seguimiento de los avances en la creación de estos materiales 


\section{Resultados}

Para la primera fase del estudio se creó un grupo de expertos. Las decisiones de este grupo configuraron los diversos bloques que debían de ser tratados. Estos fueron: FT, para alumnos de primer curso de grado; PCB, para alumnos de segundo y PCA, para cuarto y profesionales como parte de su formación de postgrado. También se decidió la configuración de los grupos focales y los profesionales del grupo de expertos que formarían parte de cada uno de los grupos focales. Además, se elaboró un estudio estadístico para evaluar estas OVA y que se llevará a cabo en el curso 2021-22. El diseño que se ha propuesto para este estudio es el de un estudio de intervención donde se evaluará haciendo uso de dos grupos, uno con las nuevas OVA en RA/RV y otro según técnica convencional al uso. La variable principal de resultados será la variación en el nivel de conocimientos y la satisfacción con la formación recibida. Tanto el grupo de experimento como el grupo de control (elegidos aleatoriamente) pertenecerán al mismo curso/grupo. Se controlará la influencia de variables externas, mediante un esquema pre-postest para valorar el nivel de conocimientos.

Así, el grupo de expertos ha decidido los bloques a tratar, ha designado los profesionales referentes para la segunda fase del proyecto, ha diseñado el estudio que se llevará a cabo para probar la herramienta y además ha adquirido los materiales inventariables necesarios para poder llevar a cabo el proyecto. Además, este grupo ha coordinado una serie de reuniones grupales (una mensual) a lo largo de todo el proyecto.

Para la segunda fase del estudio se han creado diversos grupos de discusión: el grupo de FT, el grupo de PCB y el grupo de PCA. Este último, dado su nivel de complejidad, ha precisado la división en tres subgrupos: PCA-trans, PCA-quirófano y PCA-salud mental.

Los OVAs que se muestran a continuación y son resultados de nuestro trabajo, pueden ser consultados en la página web https://sites.google.com/ull.edu.es/raviten.

\section{Resultados en forma de los OVA obtenidos por el grupo de Fundamentos Teóricos (FT)}

Se han generado dos OVA. Un póster interactivo haciendo uso de RA donde se reflejan las principales escuelas de pensamiento que dan lugar a las teorías y modelos de enfermería. Un video de $360^{\circ}$ donde se recrea un momento histórico con un valor didáctico muy importante para el alumnado (se recrea una conversación donde Florence Nightingale narra las bases sobre las que se fundamenta el actual concepto de cuidado de pacientes y de hospitales).

\section{Resultados en forma de los OVA obtenidos por el grupo de Práctica Clínica Básica (PCB)}

Se ha generado un póster haciendo uso de la RA donde se recogen las principales técnicas que un alumno debe aprender para tener contacto con pacientes hospitalizados. El póster recoge tanto un video de realización de cada una de las seis técnicas que trata como un documento (en formato presentación) de los diagnósticos e intervenciones implicadas en el cuidado de los pacientes sometidos a estas técnicas. Los vídeos de las técnicas también han sido generados por el propio grupo.

\section{Resultados en forma de los OVA obtenidos por el grupo de Práctica Clínica Avanzada (PCA)}

Tal como se mencionó previamente, este grupo se ha dividido en tres subgrupos debido al mayor grado de complejidad de los contenidos de los que tratan. El funcionamiento y la distribución de estos subgrupos es totalmente análoga a la del resto de grupos de discusión.

Los OVA de estos subgrupos han sido: 
En el grupo PCA-trans se ha elaborado un póster interactivo en formato RA que recoge materiales de apoyo tanto a las personas transgénero como a los profesionales con los que interactúan (profesionales de la salud, de la educación, del derecho...). El póster refleja documentos multimedia generados y difundidos a través de la plataforma OCW (2SHQ \&RXUH : DH de la Universidad de La Laguna (https://campusvirtual.ull.es/ocw/course/view.php?id=151).

En el grupo PCA-quirófano se ha generado un tour virtual en $360^{\circ}$ que transcurre dentro del área quirúrgica. El quirófano es un lugar de acceso restringido y altamente específico y tecnificado y que genera muchos temores a la persona que tiene que acudir a trabajar o a realizar prácticas en él. Este tour logra una inmersión en ese mundo quirúrgico. Las imágenes en $360^{\circ}$ sobre las que se basa, están apoyadas por múltiples imágenes, textos y videos que ayudan al estudiante a conocer el bloque quirúrgico. Ofrece además dos videos de dos cirugías: una cirugía abierta y otra por laparoscopia que buscan mitigar el bache que sufren los alumnos y los profesionales recién contratados en el área quirúrgica. El tour puede ser visualizado en la pantalla del dispositivo o haciendo uso de gafas VR para que así la inmersión sea más completa

En el grupo PCA-salud mental se ha generado una app en la que los expertos trabajan diversas locuciones que ayuden a personas sin ninguna patología mental de base a salvar problemas situacionales o a relajarse para poder mejorar su calidad de vida y bienestar. Estas locuciones se acompañan de música y de imágenes en $360^{\circ}$ que son visionadas a través de gafas VR, consiguiendo así un alto grado de concentración y de inmersión en las palabras que se locutan.

Para apoyar a estos grupos de discusión específicos, ha sido necesario crear un grupo de apoyo que se encarga de facilitar los recursos y el soporte técnico que cada uno de los grupos de discusión necesita. Este grupo de apoyo también mantiene los repositorios con los OVA que se van generando y los subproductos de estos OVA (vídeos, audios, textos, fotos...). El esquema de los resultados obtenidos se muestra en la figura 1. 


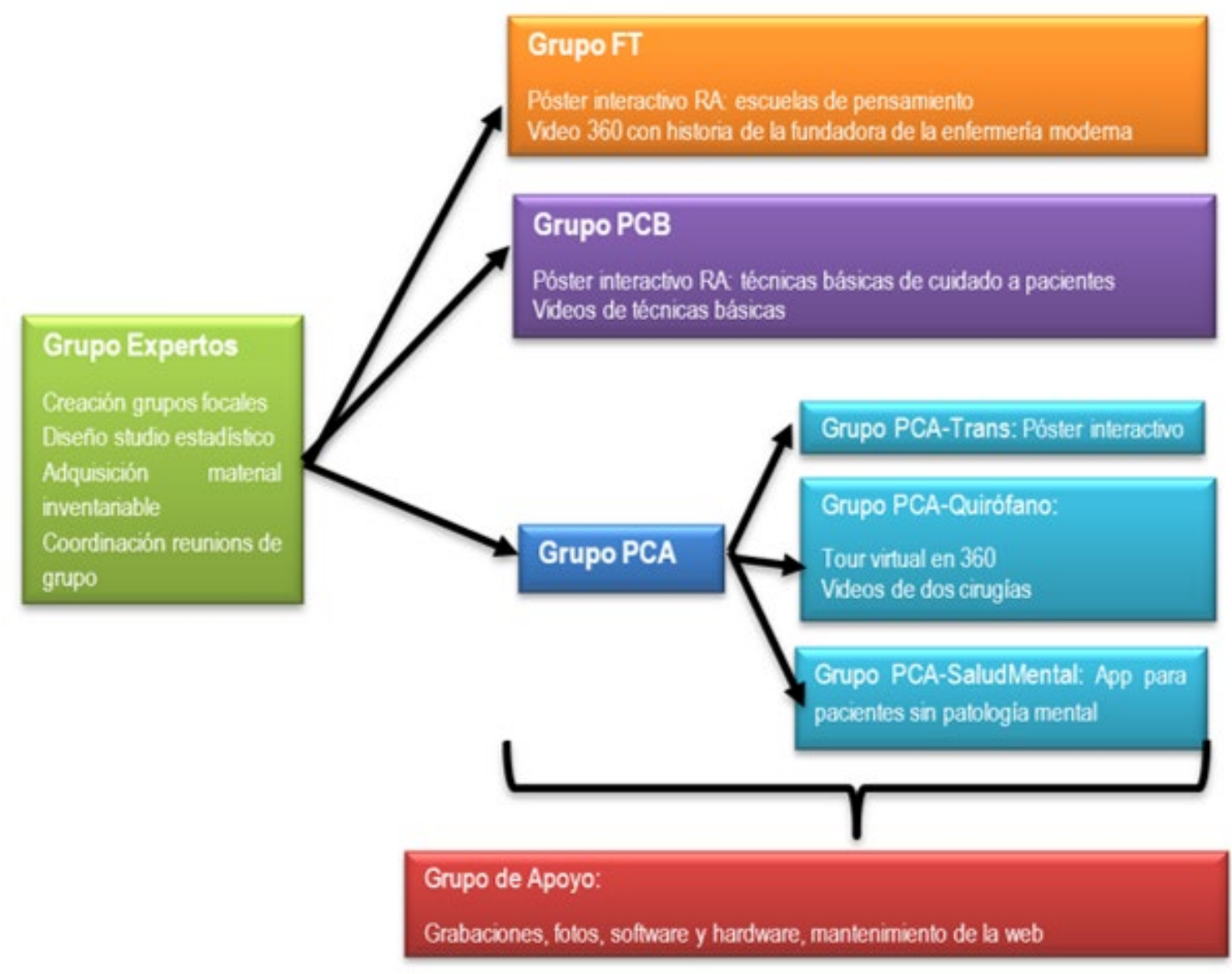

) $L X W D \square[(V X H P D G H U H X Q D C R V$

\section{Conclusiones}

Este proyecto tiene como objetivo generar materiales educativos en formato OVA que puedan ser utilizados por los alumnos de ciencias de salud y favorecer su aprendizaje. Específicamente, los contenidos generados están dirigido a alumnos de enfermería. Se ha diseñado un total de seis OVAs distribuidos en tres grandes bloques de contenidos: Fundamentos Teóricos (FT), para alumnos de primer curso de grado, Práctica Clínica Básica (PCB) para alumnos de segundo y Práctica Clínica Avanzada (PCA) para alumnos de cuarto y de postgrado.

Cuando se consultan materiales generados haciendo uso de RA/RV muchas veces no resultan satisfactorios porque hay una brecha importante entre los diseños y la realidad. Por ejemplo, no es lo mismo ver un pulmón en una cirugía que ver una recreación tridimensional de un modelado de un pulmón. Este ultimo es un trabajo ímprobo de diseño y computación y sin embargo tiene un impacto limitado en la realidad de la enseñanza de las ciencias de la salud. En estos casos, una imagen real es mejor que un objeto 3D. Los OVAs generados en este proyecto aprovechan la oportunidad de la multidisciplinariedad de sus autores para tener acceso a imágenes de la vida real tal y como van a ser encontradas por nuestros estudiantes.

Otro de los elementos positivos a destacar de los materiales generados es que son productos fácilmente transferibles a cursos siguientes y a otras facultades de ciencias de la salud, generando así sostenibilidad en el producto obtenido y la posibilidad de ampliar el número de estudiantes que se pueden beneficiar de los mismos. 
Algunos de las OVAs han sido generados sin ningún tipo de comparador o recursos ya existente, mientras que en otros hemos podido encontrar proyectos con alguna similitud. Este es el caso fundamentalmente del tour de quirófano y de la app de salud mental. En el caso del tour de quirófano, hay algunas experiencias de tours virtuales y de simulaciones de realidad basadas en simulación (https://storage.netfs.com/hosting/2727323/380/index.htm). Sin embargo, estas páginas no corresponden al mundo real, al que se espera cuando el alumno o el nuevo profesional acuden a quirófano. Esto genera que se produzca un gap entre lo simulado y lo que sucede en la realidad. El hecho de crear materiales educativos con grupos multidisciplinares hace que se conozcan en profundidad las necesidades reales de atención a pacientes. Así, el tour de quirófano responde a una visión de un quirófano real y no simulado y con estaciones e imágenes reales. El apoyo de la realidad virtual lo hace mucho más inmersivo y permite al alumno investigar sin miedo a dañar al paciente por falta de pericia. También le permite conocer un circuito complicado y tecnológico, en el que las limitaciones de acceso y tiempo de visita están presentes. Otro OVA ha sido la app de salud mental. En la red hay varias aplicaciones que tienen como finalidad facilitar estrategias de relajación, pero no responden a nuestro objetivo de trabajar no solo la relajación por estrés sino la fatiga emocional y la gestión del miedo. Además, en este recurso, la persona que lo utiliza puede seleccionar las imágenes y sonidos con los que acompañar la locución, de manera que se produzca un mejor ajuste a las necesidade3s y preferencias individuales. Para el resto de los OVAs no se ha encontrado un recurso comparable. Los materiales docentes generados en este proyecto se han desarrollado específicamente para cubrir unas necesidades concretas de formación. En este sentido, han partido de la necesidad de explicar y detallar un conjunto de contenidos especializados, han sido creados a medida de nuestras necesidades formativas y se han diseñado en concordancia con las competencias definidas en la titulación. La enfermería es una profesión regulada y cuyos descriptores de contenidos y competencias están recogidas ministerialmente. Esto nos ha llevado a adoptar esta nueva metodología para explicar contenidos específicos y de obligado cumplimiento.

Tal como afirma la literatura, la innovación educativa en el área de las ciencias de la salud debe de ser primordial para la mejora de la práctica (Ruiz Cerrillo, 2019). Estudios similares al que planteamos han encontrado como resultados que el alumnado mejoró su proceso de enseñanza-aprendizaje mediante el aumento de la motivación hacia el desarrollo de competencias cognoscitivas; además del aumento de la motivación por aprender (Ruiz Cerrillo, 2019). Existe una fuerte influencia de la tecnología digital ya que los estudiantes de enfermería transforman la información en conocimiento para adquirir actitudes y habilidades. Los OVAs se comportan como un método dinámico, constructivo e innovador que se adecua a las necesidades de aprendizaje de los estudiantes, acercándolos al entorno real del futuro profesional de enfermería, donde coloca en práctica lo que aprende, siendo capaz de tomar decisiones, superando las dificultades de la práctica. Esto hace que se reduzcan errores pues se pueden repetir los ejercicios todas las veces que sea necesario hasta dominar la técnica, generando seguridad en el estudiante y el fortalecimiento de las habilidades clínicas (Sanabria, 2019). En futuros estudios, se podrá comprobar la eficacia pedagógica y motivacional de los contenidos desarrollados.

La sensación de seguridad que se genera en los alumnos al trabajar en entornos simulados donde no se puede dañar a un paciente real es un factor motivador tanto para los estudiantes de ciencias de la salud como para sus docentes. De este tipo de aprendizaje se benefician también los pacientes, puesto que eleva la calidad de la asistencia.

En resumen, las ventajas que aportan materiales como los que se presentan en este trabajo son numerosas. La RV en educación posibilita aprendizajes inmersivos, constituyéndose no en el futuro sino en el presente de la educación. 


\section{Referencias}

Badia Llobet, M., \& Espinosa Segura, L. (2018). Terapia Espejo y Realidad Virtual en pacientes con Miembro Fantasma Doloroso. Revisión de la literatura. https://repositori.tecnocampus.cat/handle/20.500.12367/41 [Consulta: 21 marzo 2021]

Boletín Oficial del Estado. (2017). Orden SSI/81/2017, de 19 de enero, por la que se publica el Acuerdo de la Comisión de Recursos Humanos del Sistema Nacional de Salud, por el que se aprueba el protocolo mediante el que se determinan pautas básicas destinadas a asegurar y proteger el derec. In (pp. 8277-8289). https://www.boe.es/diario boe/txt.php?id=BOE-A-2017-1200 [Consulta: 22 de abril de 2021]

Cadavieco, J. F., \& Sevillano, M. Á. P. (2017). La producción científica sobre Realidad Aumentada, un análisis de la situación educativa desde la perspectiva SCOPUS. ( GPHF, 6(1), 39-61. https://www.uco.es/ucopress/ojs/index.php/edmetic/article/view/5807 [Consulta: 24 de mayo 2021].

Calderón, S; Bournissen, Jm. Y Tumino, C. (2019). "La realidad virtual y su impacto en el aprendizaje" en XXV Congreso Argentino de Ciencias de la Computación (CACIC) (Universidad Nacional de Río Cuarto, Córdoba, 14 al 18 de octubre de 2019). http://sedici.unlp.edu.ar/handle/10915/90933 [Consulta: 26 de marzo de 2021].

Calderón, S. J., Tumino, M. C., \& Bournissen, J. M. (2020). Realidad virtual: impacto en el aprendizaje

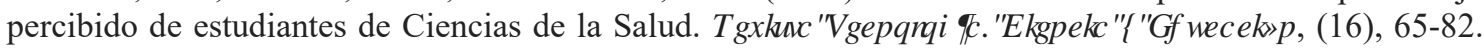
http://hdl.handle.net/20.500.12226/331 [consulta: 17 de abril 2021].

Corcó, J. (2008). La misión de la universidad en el Espacio Europeo de Educación Superior. 5HMLD 8 QIYHWWDUD( XURSHD. http://hdl.handle.net/20.500.12328/1534 [Consulta: 28 de abril de 2021].

Crespo, M. D. M. I., \& Martí, L. S. (2020). Efectividad de la realidad virtual inmersiva en el manejo del dolor del paciente quemado: una revisión sistemática. - RXLQDORIDO 2 9( $\square D Q Q G 7 K H D S H X W F] 6 F H Q F H ~ 2(2)$. https://jomts.com/index.php/MOVE/article/view/29 [Consulta: 14 de mayo de 2021].

Figuerola, S. R. (2018). Realidad virtual para superar los miedos. 5HLWDGHHQH HP HDI QWDXGP HQWDQ (11), 30-34. https://dialnet.unirioja.es/descarga/articulo/6852590.pdf [Consulta: 11 de abril de 2021].

Johnson, L., Becker, S. A., Cummins, M., Estrada, V., Freeman, A., \& Hall, C. (2016). NMC horizon report: 2016 higher education edition (pp. 1-50). The New Media Consortium.

Levy, F., Rautureau, G., \& Jouvent, R. (2018). La terapia por realidad virtual en el tratamiento de los trastornos de ansiedad1.90; HUH 51. https://www.researchgate.net/profile/JoaquinAsiain/publication/326158207_Realidades_virtuales en_Salud_Mental/links/602e6bab4585158939b3273 2/Realidades-virtuales-en-Salud-Mental.pdf \#page $=52$ [Consulta: 3 de mayo de 2021].

López, B. G., Pérez, C. P., Rodríguez, M. Á. J., Hervás, N. M., \& Beut, J. A. G. (2017). Métodos centrados en el aprendizaje, implicación del alumno y percepción del contexto de aprendizaje en estudiantes universitarios. ( GXFDFlyQ ; ; , 20(2), 161-187. https://www.redalyc.org/pdf/706/70651145007.pdf [Consulta: 19 de abril de 2021].

Martín Garcés, A. (2019). El uso de la realidad virtual para el diagnóstico y tratamiento de Trastornos de la Conducta Alimentaria: Una Revisión Sistemática. https://ddd.uab.cat/record/217995 [Consulta: 6 de mayo de 2021].

Medina, E. U., Barrientos, S. S., y Navarro, F. I. (2017). El desafío y futuro de la simulación como estrategia de enseñanza en enfermería., QYHWJDFlyQ HQ (GXFDFyQ PpGFD, 6(22), 119-125. https://www.sciencedirect.com/science/article/pii/S2007505717301473 [Consulta: 21 de abril de 2021].

Nicol, D., \& Pilling, S. (Eds.). (2005). Changing architectural education: Towards a new professionalism. Taylor \& Francis. p. 265-273.

Ramírez, A., Benítez, S. J., Y Gómez, M. A. (2017). La acrofobia y su tratamiento a través de realidad

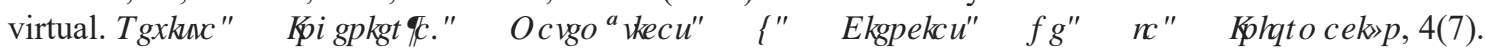
http://ojs.urepublicana.edu.co/index.php/ingenieria/article/view/346 [Consulta: 8 de mayo de 2021]. 
Rué, J. (2004). La convergencia europea: entre decir e intentar hacer. 5HILD LQUAXQIYHUWDD GH ) RLPDFlyQ GHD 3 LRIHRLDR, (49), 39-60. https://dialnet.unirioja.es/servlet/articulo?codigo=1057094 [Consulta: 4 de marzo de 2021].

Rué, J. (2007). Enseñar en la Universidad: El EEES como reto para la Educación Superior (Vol. 16). Narcea Ediciones. p. 23-25.

Ruiz Cerrillo, S. (2019). Enseñanza de la anatomía y la fisiología a través de las realidades aumentada y virtual. , QQRYDFlyQ HGXFDUWD (México, 57-76. http://www.scielo.org.mx/scielo.php?pid=S1665-26732019000100057\&script=sci_arttext [Consulta: 24 de abril de 2021].

Sanabria, M. L. V., \& Lendínez, A. J. C. (2019). Construcción y validación de un Objeto Virtual de Aprendizaje (OVA) en la administración de medicamentos en pacientes pediátricos. 5 HILWD 9

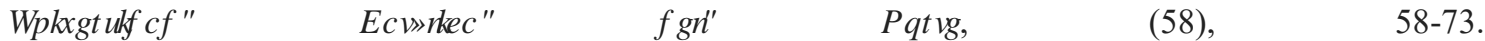
https://revistavirtual.ucn.edu.co/index.php/RevistaUCN/article/view/1086 [Consulta: 12 de abril de 2021].

Trujillo, M. H. S., Patarroyo, C. P. C., Velásquez, N. R., Parra, M. L., Burbano, P. A. G., \& Martínez, C. A. P. (2019). Interpretación del hemograma automatizado a través de un Objeto Virtual de Aprendizaje (OVA): Descripción de la experiencia. ( QWDPDOR, 15(2), 276-285. https://revistas.unilibre.edu.co/index.php/entramado/article/view/5731 [Consulta: 11 de abril de 2021].

Valbuena, S. M., Fernández, J. A. F., \& Fernández, I. F. (2019). Eficacia de una intervención enfermera con realidad virtual en Urgencias Pediátricas: un ensayo clínico aleatorizado. 5НИИМНQЮ Р БtD\&1/, 11(1), 84-94. http://www.revistaenfermeriacyl.com/index.php/revistaenfermeriacyl/article/viewFile/244/213 [Consulta: 3 de mayo de 2021].

Viedma Cervigón, A. (2017). Tratamiento a través de Internet y Tratamiento con Realidad Virtual para el Trastorno de Pánico y la Agorafobia: una revisión narrativa. http://repositori.uji.es/xmlui/handle/10234/169394 [Consulta: 7 de abril de 2021].

Yániz, C., \& Villardón, L. (2008). 3 (DQIIFDUGHMHFRP SHAQFIDVSDWDSLRP RYHUHODSUHQG]DVA(Vol. 12). Universidad de Deusto. 